\title{
Incremental benefit in correlation with histology of native T1 mapping, partition coefficient and extracellular volume fraction in patients with aortic stenosis
}

\author{
Vassilis Vassiliou ${ }^{1,2^{*}}$, Katharina Wassilew ${ }^{3}$, Tamir Malley ${ }^{1}$, Claire E Raphael ${ }^{1,2}$, Rebecca S Schofield ${ }^{4}$, Kevin Kirby', \\ Alex D Bowman ${ }^{7}$, Karen Symmonds ${ }^{1}$, Bruce S Spottiswoode ${ }^{6}$, Andreas Greiser ${ }^{5}$, lain Pierce ${ }^{1}$, David Firmin ${ }^{2,1}$, \\ Peter Gatehouse ${ }^{1,2}$, Dudley J Pennell ${ }^{1,2}$, Sanjay Prasad ${ }^{1,2}$ \\ From 19th Annual SCMR Scientific Sessions \\ Los Angeles, CA, USA. 27-30 January 2016
}

\section{Background}

We investigated the histological correlation of native $\mathrm{T} 1$ maps, partition coefficient and extracellular volume fraction (ECV) using an 11 heart beat (11 HB) MOLLI for identification of overall burden of fibrosis.

\section{Methods}

Ten patients ( 8 male, age $73 \pm 7$ years; all in sinus rhythm, 2 with ventricular ectopy) with severe aortic stenosis (3 with coexisting coronary artery disease) scheduled for surgical aortic valve replacement underwent CMR on a $1.5 \mathrm{~T}$ scanner (MAGNETOM Avanto, Siemens Healthcare, Erlangen). The 11HB MOLLI sequence (Siemens investigational prototype WIP 448B) was acquired before and 15 minutes post $0.1 \mathrm{mmol} / \mathrm{kg}$ gadolinium administration. Incorporating hematocrit results from the same day. This allowed native T1 maps, partition coefficient and ECV calculation. Images were obtained twice at end diastole at basal, and twice at mid left ventricular level. The average of all measurements was used to calculate ECV using the standard formula Partition Coefficient $=[(1 /$ T1 myocar dium post contrast-1/T1 myocardium native $)] /[(1 / \mathrm{T} 1$ blood post contrast-1/T1 blood native)] with $\mathrm{x}(1-\mathrm{HCt})$ for ECV. Similar regions of interest were drawn in the septum at both levels for T1 values. Intraoperatively, trucut biopsies were taken from the left ventricular apical anterior/ lateral wall through the epicardium to allow histological characterization of the full myocardial wall, and fixed in

${ }^{1}$ CMR, Royal Brompton Hospital, London, UK

Full list of author information is available at the end of the article warm buffered formalin. Histological analysis of formalinfixed paraffin-embedded, transmural myocardial biopsies of the left ventricle was performed on hematoxylin/eosin and Picrosirius red-stained 3-micron-thick sections by a blinded experienced cardiac pathologist. Images were analysed using a purpose-built software (Nikon NIS elements $\mathrm{BR}$ ) on a NIKON Eclipse light projection microscope to determine the extent of overall and reactive interstitial fibrosis, which was expressed as collagen volume fraction (\%) per square millimetre.

\section{Results}

Native T1 mapping, partition coefficient and ECV all correlated with histologically measured fibrosis. However, native T1 mapping showed the least accuracy (panel $A, R^{2}$ $=0.42$ ) and ECV showed the highest accuracy (panel $B, R^{2}$ $=0.83$ ). Partition coefficient was more accurate than native T1 mapping but only very marginally less so than ECV (panel C, $\mathrm{R}^{2}=0.80$ ).

\section{Conclusions}

These results suggest that native T1 mapping is less accurate than partition coefficient and ECV for overall fibrosis. Therefore, post gadolinium images to enable calculation of partition coefficient and ECV should be routinely obtained to increase accuracy.

\section{Authors' details}

${ }^{1}$ CMR, Royal Brompton Hospital, London, UK. ${ }^{2}$ National Heart and Lung Institute, Imperial College London, London, UK. ${ }^{3}$ Cardiac Pathology Unit, Department of Cardiothoracic and Vascular Surgery, Deutsches Herzzentrum 


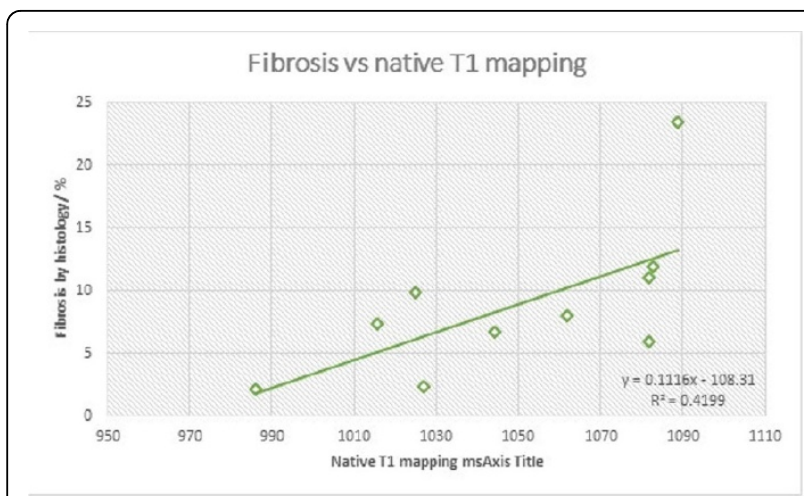

Panel A: Correlation between CMR native T1 mapping and histologically identified fibrosis, $R^{2}=0.42$

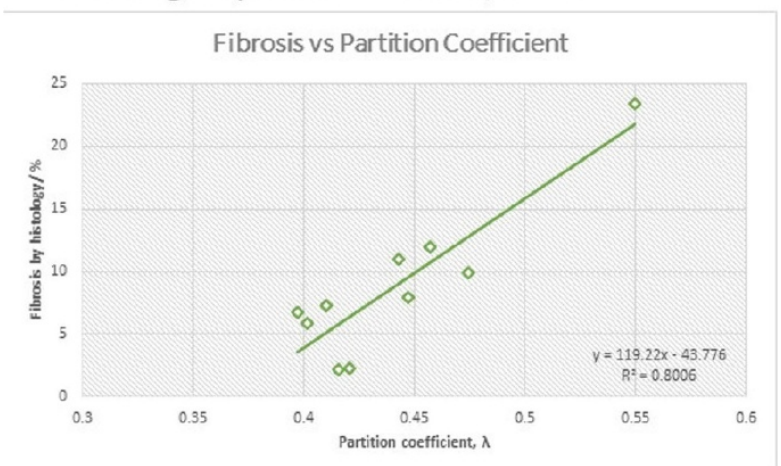

Panel B: Correlation between CMR Partition coefficient (based on native and post Gd T1 maps) and histologically identified fibrosis, $R^{2}=0.80$

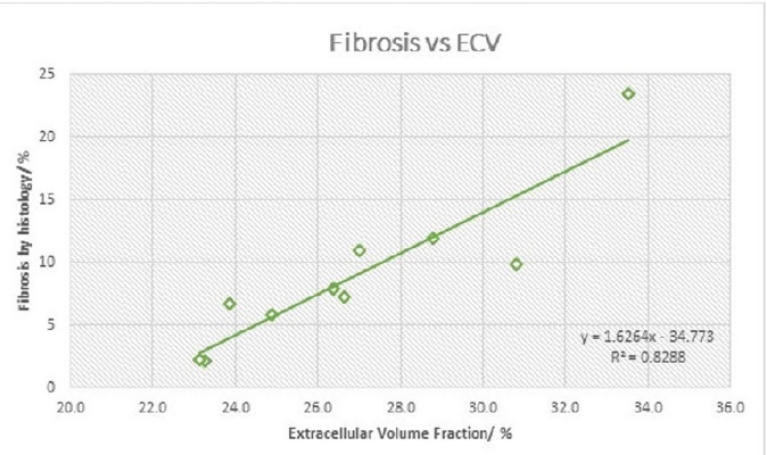

Panel C: Correlation between CMR ECV and histologically identified fibrosis, $R^{2}=0.83$

Figure 1 Correlation between native T1 and histological fibrosis (Panel A, top); correlation between partition coefficient and and histological fibrosis (Panel B, middle); and correlation between ECV and histological fibrosis (panel C, bottom). Both partition coefficient and ECV perfomred better than native T1 maps alone.

Berlin, Berlin, Germany. ${ }^{4}$ Imaging, UCL, London, UK. ${ }^{5}$ Siemens Healthcare, Erlangen, Germany. ${ }^{6}$ Siemens Medical Solutions USA, Inc, Chicago, IL, USA. ${ }^{7}$ Department of Histopathology, Royal Brompton Hospital, London, UK.

Published: 27 January 2016
doi:10.1186/1532-429X-18-S1-048

Cite this article as: Vassiliou et al:: Incremental benefit in correlation with histology of native T1 mapping, partition coefficient and extracellular volume fraction in patients with aortic stenosis. Journal of Cardiovascular Magnetic Resonance 2016 18(Suppl 1):O48.

\section{Submit your next manuscript to BioMed Central and take full advantage of:}

- Convenient online submission

- Thorough peer review

- No space constraints or color figure charges

- Immediate publication on acceptance

- Inclusion in PubMed, CAS, Scopus and Google Scholar

- Research which is freely available for redistribution
C Biomed Central 